\title{
EL DIÁLOGO CON EL PÚBLICO Y LOS ESPACIOS REALES Y DE MARAVILLA EN CASOS PRODIGIOSOS Y CUEVA ENCANTADA DE JUAN DE PIÑA ${ }^{1}$
}

\author{
ANGela Fabris \\ Alpen-Adria-Universität Klagenfurt \\ angela.fabris@aau.at
}

\begin{abstract}
asos prodigiosos y cueva encantada (Madrid: Imprenta del Reino, 1628) es una novela escrita por Juan Izquierdo de Piña durante el lustro 16241629, en el que este autor conquense, conocido por su amistad con el Fénix, se dedicó a la redacción de algunas novelas de diferente medida y éxito. Es, además, su obra más popular, según lo atestiguado por Cotarelo y Mori que, en el estudio biográfico y crítico que introduce la edición publicada en 1907, califica a su autor como «de orden secundario», aunque subrayando sus «cualidades de invención y originalidad» (XLI).

Se debe probablemente a su rango inferior en el universo literario la pervivencia de pocos datos biográficos, entre los cuales se cuenta la noticia, incluida en el Laurel de Apolo (Vega 2007: 180-181), de su lugar de nacimiento, Buendía, en la provincia de Cuenca (hacia 1566). Después de un temprano traslado a Madrid, residió probablemente de forma estable en la corte, ocupando los cargos de Escribano de Provincia y de Notario del Santo Oficio ${ }^{2}$. Su permanencia en este ambiente se refleja también en el texto en cuestión, que con frecuencia - sobre todo en la segunda mitad - se acerca, con mayor o menor aproximación, al mundo cortesano.

La actividad literaria de Piña, situada en una fecha tardía de su vida, empieza con una colección de relatos cortos - Novelas ejemplares y prodigiosas historias

\footnotetext{
1 Este trabajo se inscribe en el marco del Proyecto I+D+i del MINECO Novela corta del siglo xvII: estudio y edición (y II) (FFI2013-41264-P).

2 Para su biografía véanse Cotarelo y Mori (1907: IV-XLII), Formichi (1967: 106-115), Fonquerne (1976: 127-134), García de Dini (1980: 99-116), Ripoll (1991: 90-93) y Bonilla Cerezo (2010: 76-78).
} 
de 1624- a la que seguirán cuatro libros más —Varias fortunas (1627), el ya citado Casos prodigiosos y cueva encantada (1628), la Segunda parte de los Casos prodigiosos (1629) y el Epítome de la primera parte de las fábulas de la antigüedad (1636) - , que denuncian una clara preferencia por el género híbrido de la novela corta barroca, y su actitud abierta y curiosa frente a distintos géneros de letras. Confirma este juicio el citado Casos prodigiosos y cueva encantada, en el que se asiste a una mezcla de patrones narrativos distintos, a los que se suma la presencia del viaje y de un universo móvil entre espacios reales e imaginativos. Tal variedad nos muestra la preferencia de Piña por la esfera del deleite ${ }^{3}$, de acuerdo con la conocida fórmula horaciana rebautizada por Cervantes en términos de «enseñar deleitando» ${ }^{4}$. En Casos prodigiosos y cueva encantada, de hecho, la intención didáctica no se menciona ni en el Prólogo, ni en el recorrido textual, en el cual las digresiones proponen principalmente comentarios al hilo de la materia o del desarrollo de esta obra de ficción.

La cuestión del deleite se relaciona también con el público de lectores u oyentes a quienes se dirige Piña. Aunque en la novela se asiste solo raras veces al establecimiento de un contacto directo entre el narrador y sus receptores, estas apelaciones al lector permiten presuponer la dificultad del autor a la hora de individualizar precisamente al público al que dirigirse, a propósito de «un género a caballo entre la corte y los inicios de la 'burguesía'» (Bonilla Cerezo 2010: 20). Lo atestigua la voz del narrador que, en el prefatio, después de haber calificado el libro como «de casos prodigiosos y sutiles pensamientos» (Piña 1907: 10) con una indicación de orden metatextual, precisa: «El prólogo no dice a quién, porque no sabe a quién» (10). Es evidente, en este sentido, la presencia en el texto preliminar de una ansiedad comunicativa hacia un destinatario todavía indefinido; una manera de establecer un contacto abierto, en su nivel máximo, con el público y la consiguiente dificultad de aclarar las condiciones básicas del diálogo con él.

No se puede hablar solamente de una incapacidad para identificar a los receptores; a ella hay que añadir un elemento más, es decir, la tentativa por parte del narrador, en los esporádicos casos en los que se dirige a los lectores, de prevenir eventuales oposiciones o desconfianzas. Por ejemplo, ya al principio, cuando, después de haber presentado a don Juan que, de niño, había aprendido astronomía y astrología, además de italiano, francés e inglés, anota: «Si el que lee dice que es fábula saber un hombre en pocos años tantas ciencias, y que no lo siendo sería prodigio, se responde que, como primera aventura prodigiosa, no dirá que desdice del asunto» (19).

\footnotetext{
Véase Formichi (1967: 101).

4 Véanse, sobre este particular, las palabras del Canónigo sobre los libros de caballería en la Primera parte del Quijote (I, 47).
} 
Más que una clara prueba de la materia prodigiosa del relato, esta inserción representa una directa emanación de quien, dándose cuenta de la existencia de un género afectado por un cierto número de estereotipos, intenta prevenir las críticas o defender su labor como novelista. Es lo que sucede cuando el narrador, en Casos prodigiosos y cueva encantada, a propósito del título, observa: «Buen título dio al libro [se refiere a las aventuras prodigiosas que está contando el protagonista], que dicen es más difícil que hacerle» (251).

Un pasaje, este, en el que se subraya la calidad de las historias insertadas, apelando a un término que reaparece con frecuencia, tanto en el propio título como en el desarrollo de la aventura de don Juan, y que se caracteriza por una irrupción de orden metatextual que, sin señales distintivas, interfiere en el diálogo de los dos protagonistas. En este caso, además, el comentario se sitúa exactamente en un momento en el cual la materia parece adolecer de cierta incoherencia o de una forma de desequilibrio. La historia, en efecto, consiste en el viaje de don Juan con su amigo Ricardo, ambos movidos por la intención de dar una vuelta por el mundo. Se describe así el camino hasta Irún, después del cual se produce un claro cambio de dirección porque tiene lugar el encuentro decisivo entre el protagonista - que se ha quedado solo- y un joven francés, el supuesto don Carlos. A partir de entonces, los dos prosiguen juntos hasta París, donde don Juan descubre cómo, en realidad, detrás del disfraz del mancebo, se esconde madama Blanca. Finalmente, se asiste a la consumación del amor entre ambos, acompañada de la narración de la aventura de don Juan en la cueva encantada.

La necesidad de confirmar la validez del título se advierte exactamente en el momento en el que el recorrido textual pierde su linealidad, después del inicio del cuento de la aventura prodigiosa en la cueva, que se desarrolla, en diferentes etapas, durante los encuentros privados entre el protagonista y madama Blanca. Si Piña, por un lado, nunca se preocupa de subrayar la finalidad moral del relato, por el otro, sostiene con frecuencia la supuesta verdad de lo que está contando; por ejemplo cuando, después del socorro - por parte de don Juan - a una dama que un galán había intentado violar, afirma: «Parece cuento de libro de caballerías; cierto que no lo es, sino verdadero suceso, este y los demás del valiente don Juan, sabidos con la debida certidumbre para darlos a la estampa» (32).

El hecho de enlazar la materia caballeresca con el principio de la supuesta verosimilitud de la fábula parece adquirir el estatus de requisito esencial para la impresión. Además, el recurso a declaraciones de este tipo puede ser también una manera de prevenir las críticas fruto del gusto viciado de los lectores de la época, hartos - probablemente- de leer y releer siempre las mismas hazañas. Así, durante la cena de don Juan y del supuesto don Carlos, se inserta un comentario llamado a criticar las convenciones ligadas a lo pastoril y a lo caballeresco: «Cenaron regalos y cuidados muchos. Esto es, a propósito, no como los libros de los 
pastores y de las caballerías, que no comían ni dormían, y esta fue la causa de haberlos tenido por fabulosos» (121-122) .

Las inserciones metatextuales desempeñan, en este como en otros casos, el papel de mantener despierta la atención del destinario, remarcando la distancia frente a los típicos enredos pastoriles y caballerescos, que por entonces habían perdido buona cuota de credibilidad entre el público. Las críticas - por otro lado- tampoco eluden la materia amorosa, aunque concebida en sentido general: «Largo va el discurso, perdone el que leyere, si tiene amor, que en tratando lo amado pocos saben darle fin» (158).

En este ámbito, la pregunta que surge espontáneamente — frente al peligro de un lector distraído - es quién leía esas novelas que — se supone- no disfrutaron de un éxito masivo. Lo más probable es presumir su difusión entre la «nueva nobleza urbana que, a pesar de la oscuridad textual, busca un elitismo cultural y no jerárquico», como subraya Bonilla Cerezo (2006: 26). Lo atestigua también el destino final de don Juan, reflejo del de sus probables receptores; justo cuando el caballero, después de haber decidido quedarse en París por amor, acepta la propuesta de madama Blanca de comprar un título nobiliario. Una posible alusión, esta, a un público de lectores sabedores de su superioridad intelectual, aunque carentes de linaje ${ }^{6}$.

Como ya he anticipado, por lo que concierne a la estructura la novela se divide en dos partes; la primera, constituida por hechos intercalados repartidos a lo largo de catorce episodios, mientras que la segunda, más amplia, gira sobre todo alrededor de la historia de amor entre el protagonista y madama Blanca, y en la descripción del viaje a la cueva encantada.

Bajo esta perspectiva, tiene sentido descifrar no solamente el material incluido en la primera mitad (o sea, su pertenencia a un ámbito específico como el pastoril, el caballeresco o el cortesano), sino de qué manera resulta incorporado en el texto, reflexionando sobre los puntos de conexión a través de los cuales se inserta cada historia. Por ejemplo, durante el viaje de don Juan con su amigo Ricardo, la mención del recorrido es la excusa que permite conceder espacio a la narración de sucesos intercalados, como el del toro Hosco (que se desarrolla «cerca del camino» $)^{7}$ o el caso de Doña Clara, que llama la atención de los «caminantes», a través de unas voces tristes y doloridas de mujer, lo que despierta «el oído atento» de don Juan (Piña 1907: 30).

\footnotetext{
Una crítica análoga se descubre en varias irrupciones de la voz narradora del Pastor de Fílida (1582) de Luis Gálvez de Montalvo, que dejan entrever cómo la materia bucólica queda expuesta a finales del Quinientos a una pérdida de homogeneidad y a una forma de cuestionamiento frente a su progresiva formalización. Veánse Montalvo (1999: 270 y 321) y Fabris (2012: 30).

6 Formichi (1976: 101).

7 Piña (1907: 25).
} 
La descripción del asesinato de don Pedro Antonio se produce también durante el camino hacia San Sebastián, cuando asistimos al encuentro con una tropa formada por alguaciles de corte y un preso. La historia es contada a los que se disponen a escuchar, mientras se intenta reparar una rueda del coche, en un sitio apartado, cerca de «una fuente que, si no secreta, murmuraba fría» (56), cual probable eco del valle decameroniano. Y el caso lo relata un escribano - proyección del mismo Piña - del que se dice que «era muy buen relator» (56). De este modo, se despliega otro nivel narrativo dentro de la historia y se inserta un narrador de segundo grado que toma directamente la palabra. Es él quien, dirigiéndose a los que le escuchan, pide disculpas por sus digresiones: «-Perdonen vuesas mercedes (dijo a los oyentes) si dilato el cuento, que más dilata el cochero el aderezo y no sé cuál dará más pronto fin» (59).

Es decir, un discurso directo del escribano (a guisa de diálogo esclarecedor) donde el paréntesis se ha de atribuir al narrador de primer grado (atestiguado por el uso de la tercera persona); prueba de cómo, de vez en cuando, interrumpiendo el relato diegético de la historia, el tejido textual se abre a la inserción de fragmentos dialogados, alusivos casi a una puesta en escena teatral.

Otra interrupción en el recorrido se cifra en la breve descripción de una lucha entre un pastor y una culebra, privada de relevancia a través de un lapidario juicio final: «don Juan y Ricardo admiraron el caso, no pareciendo novedad portentosa» (79), dado que, de historias similares, el protagonista ya había visto y leído. A renglón seguido otra consideración metatextual, relativa nuevamente a una crítica hacia el elevado número de maravillas presentes en la fábula de tantas novelas de la época, es decir, en un horizonte de espera ya cansado de tales artificios: «Hasta San Sebastián no encontraron otras desdichas a deslumbrar, que no se hallan a cada paso» (80).

Estas inserciones aseguran cómo, en el desarrollo de la primera parte de Casos prodigiosos y cueva encantada, las historias intercaladas parten en su mayoría del camino de don Juan, de tal forma que el recorrido se convierte en elemento estructural unificador, por cuanto el narrador, en relación a los lugares donde se detiene el protagonista, nos proporciona solamente datos escuetos. Se trata, en síntesis, de una elección que, oponiéndose «a la lejanía maravillosa de los libros de caballerías» (Arredondo 1996: 146), se refiere a Castilla, privilegiando el requisito de la proximidad geográfica.

Paralelamente a estos elementos que nos remiten a un espacio conocido, asoman interpolaciones de diferente carácter: por ejemplo, la descripción de la quinta y de los cuadros de don Antonio de Sotomayor, donde se aposenta don Juan para visitar a un personaje que había conocido y frecuentado en la corte, cual universo de referencia. En el mismo panorama se deslizan también episodios como el de las conclusiones de San Sebastián, que revelan un precioso cuadro de las disputas 
culturales de la época, el encuentro picaresco entre Polonia y don Carlos (contado por este último, bajo su disfraz masculino) y la terrible historia de Duarte y Aurelia, a quienes se ahorca - aunque ya muertos- por haber matado por avidez, ignorándolo, a su propio hijo. Este último episodio se desarrolla en suelo francés, al otro lado de la frontera, y se caracteriza por la dialéctica en acción entre un espacio familiar y otro (en este caso el joven volvía de México) de donde quien regresa, lo hace convertido en un extraño.

Todo este conjunto se traduce, en la primera parte, en el desarrollo de una novela «de itinerario» (García Berrio 1989: 433), fortificada a través de un elemento esencial: el de la familiaridad del público de la época con el paisaje español, y el paralelo desarrollo - durante el recorrido- de una serie de aventuras que neutralizan la percepción de una alteridad relacionada con lugares lejanos y desconocidos.

Resulta claro, de esta manera, cómo el espacio —en sus diferentes manifestaciones - tiene su demostrada importancia en la novela. Lo subraya la presencia de lugares simplemente nombrados (Laspéras 2012: 17) o sujetos a una cierta economía descriptiva dentro del texto, y su capacidad de evocar sentidos específicos en la dimensión imaginativa del lector. Me refiero a la importancia atribuida —en el plano discursivo de la novela- a la evocación de espacios, que nos lleva a hablar - en consonancia con las investigaciones de Fernando Rodríguez de la Flor (2010: 162) - de una «territorialización de la literatura». En este ámbito —por ejemplo- se sitúan las alusiones a Salamanca, ciudad letrada por excelencia, que se dirigen hacia el horizonte de espera de los lectores de la época, hábiles para colocarlas dentro de un mapa específico, es decir, el del campo letrado, que permite atribuir una cualidad más al perfil del joven protagonista (Rodríguez de la Flor 2010: 165).

Paralelamente, se pueden establecer algunas diferencias de orden espacial, a partir del predominio ideológico del núcleo urbano que sustenta la novela; un eje — más o menos visible - que nos acerca a un rasgo típico de las Ejemplares, en las cuales las descripciones «muestran una clara preferencia por lo urbano frente a lo rural» (Arredondo 1996: 147). De hecho, en una novela con una intriga de carácter amoroso, es lógico reconocer el papel desempeñado por un mundo urbano (Formichi 1967: 101) y por una sociedad protoburguesa situada en dicho universo. Esto se advierte ya en la descripción del protagonista, don Juan Bernardo, presentado al principio del texto bajo la denominación de «ilustre caballero» y «aventurero» (Piña 1907: 13), al resaltar su disposición a viajar y su pertenencia a un ambiente cortesano y urbano al mismo tiempo, como atestiguan sus datos biográficos: «don Juan Bernardo, de noble familia, de alentado y nunca ocioso ingenio, de aquellos divinos de la insigne y más que Nápoles bella Madrid, patria dichosa de los católicos y faustos Reyes de la española Monarquía de Filipo IV» (13). 
Aunque la ascendencia urbana se concreta, en esta cita, solamente en un sintético panegírico de la ciudad de la que — según las reglas propias de la descriptio urbis - se subraya su grandeza, el universo de pertenencia resulta claramente definido, dado que es exactamente en él donde se sitúa la primera aventura al inicio del viaje. Exactamente cuando don Juan, al dejar Madrid al alba, «llegando a rezar a la puerta de la iglesia de San Salvador (parece que daba principios a sus aventuras), vio que de la casa más inmediata, por la espalda de la cárcel, salían tan densas nubes y furiosas llamas que, a ser en aquel infeliz lugar, creyera ver el infierno» (20).

A continuación, se suman detalles arquitectónicos de forma conjunta con la percepción vertical de un espacio elevado, cuando se describe el primer cuarto al que suben don Juan y su amigo Ricardo. De esta manera, su aventura remarca de antemano el carácter urbano de la novela, aportando, además de una serie de elementos arquitectónicos, un efecto auditivo con «la voz de las campanas de Santa Cruz» (21), cual prueba de la capacidad de Piña para recrear — de forma sintética- una escena madrileña en su conjunto ${ }^{8}$.

El itinerario pasa después por San Sebastián y su convento, con las conclusiones que don Juan ofrecerá sin mucho esfuerzo, como elemento clave para demostrar el ingenio del protagonista, hijo de la insigne Madrid, por un lado, y de la Universidad de Salamanca, por el otro (sede, esta última, que, con el convento, remite a dominios específicos del Derecho). Así se puede decir que pasado y futuro —-sobre todo con la inserción de París como destino final- están relacionados con precisos espacios urbanos, según demuestra la llegada del protagonista a la capital francesa, en su intento de «ver sus cristianísimos reyes y palacios de único renombre» (Piña 1907: 55), además de sus «calles tan largas» (142), con una mención que califica y no describe.

El espacio interviene, pues, en la definición del mismo protagonista, formado por sus estudios y su experiencia en los ritos y en la vida urbana, demostrando cómo el hombre se califica en razón de los lugares de pertenencia, donde se imbrican marcas objetivas de identidad y relación. Es lo que sucede en el caso específico del entorno de don Juan Bernardo y también —en parte por la mediación de Blanca- lo que se desarrolla en la corte parisina, con sus príncipes y damas, caballeros y doctos ${ }^{9}$.

Junto al universo urbano, también el viaje ocupa un lugar central en la novela: don Juan no solo se traslada de un sitio a otro en suelo español, sino que también se mueve de un país a otro, es decir, de Madrid a París. No por casualidad, al final, se junta con madama Blanca quien, producto de la misma inquietud, ha salido de

8 Sobre la percepción del espacio y de una serie de sensaciones visuales y auditivas véase Laspéras (2012: 27-28).

$9 \quad$ En relación al universo cortesano y urbano de la época véase Formichi (1967: 122 y 129).

Edad de Oro, XXXIII (2014), pp. 267-280, ISSN: 0212-0429 
viaje, dejando París para ir a España. Dinamismo y movimiento —elementos clave de la mentalidad de ambos- los caracterizan así frente al estatismo de Leonor, la antigua novia de Juan, que se queda en el convento a la espera de su amado, demostrando de ese modo su pertenencia a otro ámbito ideológico.

El primer aspecto del viaje real que se advierte a través del texto es el de la intencionalidad del viajero, ya que al principio se especifica:

El aventurero don Juan Bernardo determinó en el primer discurso, que despreció rudimentos, peregrinar el mundo, penetrar secretos, maravillas y sutilezas del ingenio y desvelo, no ignorar lo político más remoto, costumbres, leyes, inclinaciones, nervios para las guerras, consejos y gobiernos en las paces, materias de estado que observan las provincias y reinos, y dar (si pudiese) una vuelta al mundo por el temido estrecho de Magallanes, no temido de aquel prodigioso Palinuro español (13-14).

No le detiene el dejar en Madrid a su querida Leonor, movido por la intención de «ver a Génova por sus fábricas, y Potosí grande de sutiles y útiles materias de estado» (16): dos sedes que apuntan a la esfera de la riqueza, del comercio y de la abundancia.

El recorrido transcurre, al principio, por la geografía de Castilla, con la mención específica de términos referidos al ámbito del viaje —el sustantivo más frecuente es «caminantes»-, además de la presencia de específicas coordenadas espacio-temporales, por ejemplo con una dilación de «un cuarto de hora» (29) o la inclusión de una historia que se entrelaza después de poco más de «media legua» de camino (30). Se advierte así una actitud de carácter realista aunque, durante el trayecto, Piña nunca describa el paisaje, cuyo papel es funcional más que simplemente decorativo. Lo que se valora - a lo largo de las directrices que sigue el homo viator - es su calidad de lugares que favorecen las relaciones, como en el caso de la inserción de la historia de doña Clara, natural de Valladolid, de la que el caballero tiene noticia durante su estancia en Guadarrama, donde se afinca dos albas (lo que permite la inserción de un breve homenaje poético a sus sierras).

Se puede afirmar, en este sentido, que el espacio queda reflejado en la novela de Piña de forma poliédrica; no faltan tampoco espacios de interior como el cuarto de la galería, adornada con pinturas de Tiziano y de los más excelentes pinceles, en que el gran caballero don Antonio de Sotomayor aposenta a don Juan, o sea, un gabinete que reúne, en una colección atemporal, un conjunto de pinturas de épocas diferentes.

A estos ejemplos se suman las descripciones de los espacios privados en los que se encuentran don Juan y madama Blanca: «subieron a una sala y por ella pasaron con las luces que había en cada una, dudando la noche hasta llegar a la antecámara de la reina. [...] Entró en una sala muy cuadrada y muy grande; las 
colgaduras, ricas y frescas» (Piña 1907: 159). Son un claro ejemplo de «detallismo interior», según una tendencia típica de la novela corta española subrayada por Arredondo (1996: 149). Luego en la concurrencia de los espacios abiertos e interiores se asiste al predominio descriptivo de estos últimos, en particular durante la segunda parte de la novela.

En el itinerario real que conduce al protagonista de Madrid a París se pueden distinguir dos núcleos esenciales: la partida y el destino. Falta por completo, sin embargo, el regreso o nostos, que resulta esencial, por ejemplo, para el personaje homérico que no quiere cambiar, sino solo volver (Morosini (2013: 24-26). En verdad, al principio del viaje la intención de don Juan —después desatendida mediante un destino final diferente - era la de regresar a Madrid para reunirse de nuevo con su amada Leonor.

A lo largo de toda la novela, el autor no se limita - sin embargo- a incluir un viaje concreto con un mapa de los lugares nombrados en el texto (por ejemplo, el itinerario que prevé Irún como primer destino), sino que añade también la aventura prodigiosa vivida por el protagonista en una cueva encantada, espacio de maravilla (Cull 2003: 165 y Baquero Escudero 2003: 57-68) relevante hasta el punto de figurar en el título de la obra. En este sentido, en sus manifestaciones en la novela de Piña, el motivo del viaje se relaciona, por un lado, con una trama de enredo amoroso, a través del recorrido concreto de una corte a otra y de un espacio urbano familiar a otro similar, aunque nuevo (Colón Calderón 2001: 13) y, por otro, con un esquema iniciático: el viaje a lo desconocido dentro del cual se sitúan - aunque no se mencionan - raíces folclóricas, literarias y culturales (Arredondo 1981: 749-760). Este último aspecto se relaciona con el elemento geográfico de la cueva, cuyo esbozo, por parte del protagonista, empieza así: «Entré por malezas y selvas intricadas, [...] me fui acercando a unas terribles peñas, de quien, como del mayor artífice, estaba hecha una calle de hierba sencida, no pisada» (Piña 1907: 238-239). Sigue la llegada del protagonista al umbral de la cueva, constituido por árboles y peñas. Son las señales típicas del ingreso siempre dificultoso en un mundo desconocido, representado en sus diferentes escenas a través de descripciones tales como una «sala cuadrada de aquellos riscos y peñas, no vistos, ni aún imaginados, ignorados del mapa, libros y relaciones» (243).

Se trata de una serie de lugares propios de los espacios naturales, con malezas, un profundo valle, una selva «amena y deleitosa» (249-250) o «un lago profundo» (269). Pero también los hay próximos a los de una arquitectura urbana —cuartos, «techos dorados» (257) o la «sala de un alcázar real» (261), cual reflejo de diferentes pruebas reales y metafóricas-. El viaje a la cueva se asocia a una serie de precedentes, en particular a la aventura de don Quijote en la gruta de Montesinos (II, XXII-XXIV), como fruto de una tradición anterior que, al distorsionarse, se desvirtúa y se mantiene simultáneamente, a través de algunos 
motivos: ante todo el del acceso del protagonista a un lugar desconocido en el que se anula la frontera entre lo real y lo ilusorio. Según afirma Castillo Martínez, a propósito de la cueva que aparece en el Quijote y en algunas novelas pastoriles, esta, como lugar al que se llega con dificultad, representaría el ámbito de lo misterioso y de lo inesperado; juicio extensible a la novela de Piña, donde se suceden, unos a otros, episodios que no dependen de la razón humana. La cueva - prosigue Castillo Martínez- es el único lugar en el que se puede encontrar «el espacio de lo mágico, pero de lo mágico posible no solo al entendimiento del protagonista sino, más importante aún, al del propio lector quien, advertido de las características de estos lugares, adiestrado ya en sus descripciones, se deja llevar con agrado y sin obstáculos hasta ellos» (Castillo Martínez 2001: 472). Lo atestigua el sueño premonitorio con un «varón hermoso de ricos y preciosos vestidos» (228), que advierte al público de Piña de las condiciones especiales del lugar, al «entrar en una cueva encantada donde le llevaría su hado, en la cual había de ver cosas portentosas, no vistas ni imaginadas» (Piña 1907: 229), en un periodo de tiempo de veinticuatro horas.

Además, el viaje de don Juan a la gruta encantada es la culminación narrativa de un esquema iniciático, partiendo de la separación del mundo y del acceso a un espacio diferente y cambiante, constituido por un alcázar, palacios o lugares de la naturaleza donde se descubre al protagonista. El proceso iniciático lo conduce a una forma implícita de renacimiento, en sintonía con la sensibilidad barroca aunque, al final, a través del retrato que el protagonista entrega a madama Blanca, el viaje a la cueva parece transformarse casi en un juego cortesano. En este sentido, la iniciación finaliza en una pulsión de carácter sentimental, dado que es el amor quien orienta los actos de los protagonistas. A esto se suma una especie de prefiguración final del paraíso, en sintonía con la que se le ofrece a la vista de don Quijote, es decir, «un bello, ameno y deleitoso prado» $\mathrm{y}$ «un real y suntuoso palacio o alcázar, cuyo muro o paredes parecían de transparente y claro cristal» (II, XXIII), como subrayaron Redondo (1981: 756) y Percas de Ponseti, que asocia esta imagen al castillo interior del místico (1980: 150) ${ }^{10}$. Es lo mismo que le sucede a don Juan cuando se encuentra delante de una «casa en medio de unos prados amenos y deleitosos jardines de flores y maravillas», donde se despliega una profusión de elementos arquitectónicos tales como una puerta, unas ventanas, unas rejas, una fachada y un pórtico hasta una delantera de «cuarentas pies y doscientos de fondo» (Piña 1907: 256-257); todo un epítome de lo que es posible encontrar en un palacio real.

Este es el momento en el cual el escenario propiciado por el recorrido por la cueva prodigiosa se acerca a su concreción, ya como un conjunto de elementos

10 Todo esto se relaciona, también, con la tesis de Propp (1974: 416-417) sobre el cuento maravilloso, donde el mundo del más allá atesora, en ocasiones, palacios de cristal y fecundos prados. 
arquitectónicos bien definidos, y donde se aclara — una vez más- cómo el verdadero mundo al que Piña se refiere es aquel urbano y cortesano, prefiguración concreta del paraíso al que aspira el hombre barroco. En síntesis, se atribuye importancia al viaje entendido como movimiento, capaz de reflejar la inquietud del hombre nuevo del Seiscientos español, que se desarrolla tanto a través del itinerario por el conocido suelo patrio, cuanto del descubrimiento de otros espacios (por ejemplo, los de la vecina París, que no es sino una forma de exotismo de grado inferior, expresión de un allá no demasiado lejano). A estos espacios reales se añade la categoría de la maravilla, es decir, la cueva encantada, como expresión de un esquema iniciático alusivo a un nuevo renacer, en línea con la búsqueda del Barroco, dominado por la aspiración al cambio. En el caso de don Juan esto significa quedarse en París con madama Blanca, dejando así a Leonor, imagen de la vieja e inmanente mentalidad.

Además, a partir del momento en que se produce el encuentro con el supuesto don Carlos, el patrón del viaje se difumina lentamente, en favor del desarrollo de la materia amorosa. Solo en la última parte, la aventura recobra su importancia, aunque según un modelo distinto, el del viaje imaginario en una cueva, con elementos semejantes al reino de las sombras dibujado por Dante en la Divina Commedia, por un lado, y con unas transparentes referencias - como se ha visto- a la cueva de Montesinos, por el otro. Debajo de tal especie, se termina asistiendo a un diálogo entre una vertiente realista - los espacios urbanos públicos y privados, alusivos a una novela «de recinto» (García Berrio 1989: 436)—y una dimensión imaginativa que transforma de continuo el conjunto de lugares, esto es, la cueva encantada en la que se desarrolla.

Lo que parece esencial en la novela de Piña no es el propósito ni de hacer reflexionar, ni de educar al auditorio, y aún menos de edificarlo, sino, más bien, de entretenerlo de forma recogida. Entonces, la novela del conquense se convierte en un texto, más o menos logrado, de puro descanso, con la intención de dejar espacio tanto al universo urbano como a la geografía española, y también a lo sorprendente y lo maravilloso, a través de unas escenas cuidadosamente imaginadas. Lo que se advierte en esta novela es, además, una forma de transición mixta - a través de una suspensión de la actitud verosímil que se demanda al lector en la primera parte- en la que los escenarios prodigiosos se sitúan en un fondo de fragmentos realistas que remiten «al mundo amable, entretenido y protoburgués de los espacios urbanos» (Bonilla Cerezo 2010: 28). Así que, a propósito de los tipos de lugares que pertenecen al universo de la novela ${ }^{11}$, llama la atención que Piña intente combinar dos elementos aparentemente opuestos sin lograrlo con plenitud: el viaje ultraterreno — con una forma de aceptación tácita

11 Acerca de la capacidad para «combinar elementos aparentemente contradictorios: lo admirable con la verosimilitud», véase Spieker (1975: 42). 
por parte del público, ya acostumbrado a experiencias análogas a través del precedente de la gruta de Montesinos- y la verosimilitud de un espacio urbano, definido en sus detalles arquitectónicos y socio-culturales.

Recibido: 17/09/2014

Aceptado: 30/10/2014

\section{BiBLIOGRAFÍA}

ARREDONDO, María Soledad (1996). «Paisajes narrativos en los siglos XVI y XVII: del lugar ameno a la selva urbana». En Actas del X Simposio de la Sociedad Española de Literatura General y Comparada. Santiago de Compostela: Universidade, pp. 143-158.

BAquero Escudero, Ana L. (2003). «Los espacios de la maravilla en la novela corta áurea». En Ignacio Arellano (ed.), Loca ficta: Los espacios de la maravilla en la Edad Media y Siglo de Oro. Actas del Coloquio Internacional, Pamplona, Universidad de Navarra (abril 2002). Madrid / Frankfurt am Main: Universidad de Navarra Iberoamericana - Vervuert, pp. 57-68.

Bonilla Cerezo, Rafael (2006). «El gongorismo en la Novelas exemplares y prodigiosas historias de Juan de Piña (II)». Il confronto letterario, 45, I, pp. 25-54. (ed.) (2010). Novelas cortas del siglo XVII. Madrid: Cátedra.

Bonilla Cerezo, Rafael, José Ramón Trujillo y Begoña Rodríguez (coords.) (2012). Novela corta y teatro en el Barroco español (1613-1685). Studia in honorem Prof. Anthony Close. Madrid: Sial Ediciones.

Castillo Martínez, Cristina (2001). «“Cuevas subterráneas”, “maletas abandonadas” y otros paralelismos entre el Quijote y algunas novelas pastoriles del siglo XVII». En Antonio Pablo Vistarini Bernat (ed.), Volver a Cervantes. Actas del IV Congreso Internacional de la Asociación de Cervantistas, Lepanto 1-8 de octubre de 2000. Palma de Mallorca: Universitat de les Illes Balears, vol. I, pp. 471-478.

Colón CALderón, Isabel (2001). La novela corta en el siglo XVII. Madrid: Ediciones del Laberinto.

Cotarelo y Mori, Emilio (1907). «Introducción». En Juan de Piña, Casos prodigiosos y cueva encantada. Colección Selecta de Antiguas Novelas Españolas. Madrid: Viuda de Rico, pp. v-XLII.

Cull, John T. (2003). «Los espacios de la maravilla en los libros de pastores españoles». En Ignacio Arellano (ed.), Loca ficta: Los espacios de la maravilla en la Edad Media y el Siglo de Oro. Actas del Coloquio Internacional, Pamplona, Universidad de Navarra. Madrid / Frankfurt am Main: Universidad de Navarra - Iberoamericana - Vervuert, pp. 165-188.

FABRIS, Angela (2012). «Estrategias narrativas y socioculturales: El pastor de Fílida de Luis Gálvez de Montalvo». En Wolfgang Matzat y Max Grosse (eds.), Narrar la pluralidad cultural. Crisis de modernidad y funciones de lo popular en la novela en lengua española. Madrid / Frankfurt am Main: Iberoamericana - Vervuert, pp. 21-34.

FonQuerne, Yves-René (1976). «Quelques documents inédits sur Juan de Piña et sa famille». Caravelle, Cahiers du Monde Hispanique et Luso-Brésilien, 27, pp. 127-134. 
Formichi, Giovanna (1967). «Le Novelas ejemplares y prodigiosas historias de Juan de Piña». Lavori della sezione fiorentina del gruppo ispanistico CNR, Serie 1. Florencia: D’Anna, pp. 99-163.

(1973). «Saggio sulla bibliografia critica della novella spagnola seicentesca». Lavori ispanistici, Serie III, Mesina-Florencia: D’Anna, pp. 5-105.

Gálvez de Montalvo, Luis (2006 [1582]). El pastor de Fílida. Julián Arribas Rebollo (ed.). Valencia: Albatros Ediciones.

García Berrio, Antonio (1989). Teoría de la literatura. Madrid: Cátedra.

GARCÍA DE DiNI, Encarnación (1980). «Juan de Piña, escribano de oficio y poeta por afición». Miscellanea Filologico-Letteraria, I, pp. 99-116.

LASPÉrAs, Jean Michel (2012). «Espacios de la novela corta». En Rafael Bonilla Cerezo, José Ramón Trujillo y Begoña Rodríguez (coords.), Novela corta y teatro en el Barroco español (1613-1685). Studia in honorem Prof. Anthony Close. Madrid: Sial Ediciones, pp. 15-35.

Morosini, Roberta (2013). «I viaggi 'statici' di Pampinea. Tra false partenze e desiderati ritorni». En Isabel Colón Calderón et alii (eds.), Los viajes de Pampinea. Novella y novela española en los Siglo de Oro. Madrid: Sial, pp. 15-28.

Percas de Ponseti, Helena (1980). «La cueva de Montesinos». En El Quijote, George Haley (ed.). Madrid: Taurus, pp. 142-174.

PIÑA, Juan de (1907). Casos prodigiosos y cueva encantada. Emilio Cotarelo y Mori (ed.). Madrid: Viuda de Rico.

- (1987), Novelas ejemplares y prodigiosas historias. Encarnación García de Dini (ed.). Verona: Università degli Studi di Pisa.

Ripoll, Begoña (1991). La novela barroca. Catálogo bio-bibliográfico (1620-1700). Salamanca: Ediciones de la Universidad de Salamanca.

Redondo, Agustín (1981). «El proceso iniciático en el episodio de la cueva de Montesinos del Quijote». En Manuel Criado de Val (ed.), Cervantes: su obra y su mundo. Actas de 1 Congreso Internacional sobre Cervantes. Madrid: EDI-6, pp. 749-760.

Rodríguez de LA Flor, Fernando (2010). «Salamanca, la ciudad letrada». En Andrés Sánchez Robayna (ed.), Literatura y territorio. Las Palmas de Gran Canaria: Academia Canaria de la Historia, pp. 161-187.

VEGA, Lope de (2007). Laurel de Apolo, Antonio Carreño (ed.). Madrid: Cátedra. 


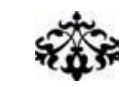

El diÁlogo CON EL PÚBLICO Y LOS ESPACIOS REALES Y DE MARAVILla

en Casos prodigiosos y CUEVA ENCANTAda DE JUAN de PIÑA

RESUMEN: Se analiza en la novela Casos prodigiosos y cueva encantada (1628) de Juan de Piña el diálogo del narrador — portavoz de una forma de ansiedad comunicativa- hacia un destinatario indefinido, en una época de transición social, por un lado, y la presencia de distintos espacios reales y de maravilla, por el otro. Dentro de la dimensión real, destaca la mención repetida del recorrido, como elemento estructural unificador de esta «novela de itinerario» en su primera parte, además del predominio ideológico del núcleo urbano, mientras en el ámbito de lo maravilloso domina el viaje a la cueva encantada, expresión de un esquema iniciático alusivo a un nuevo renacer, acorde con la búsqueda del hombre barroco, dominado por la aspiración al cambio.

Palabras Clave: Público, Espacios reales, Maravilla, Viaje, Cueva encantada, Juan de Piña.

\section{The Dialogue with the Public and the Real and Great Spaces in Casos prodigiosos y cueva encantada BY JUan DE PIÑa}

ABSTRACT: The analysis of Juan de Piña's short story Casos prodigiosos y cueva encantada (1628) centers on the dialogue between the narrator voicing a sort of communicative anxiety, and an unspecified recipient at a time of social transition, on one hand, and on the other, the presence of distinct spaces belonging to the worlds of reality and wonder. The sphere of reality abounds with references to the pathway (thought of as the gluing element of the first part of this "itinerary novel") and the ideological prevalence of the urban nucleus. The journey to the enchanted cave prevails in the sphere of wonder, representing an initiatory pattern that hints to a new renaissance, in tune with man's quest for change in the Baroque era.

KeYwords: Public, Real Spaces, Wonder, Journey, Enchanted Cave, Juan de Piña. 
Evangelina Rodríguez Cuadros (Universitat de València)

Novela cortesana, novela barroca, novela corta: de la incertidumbre al canon .9

Mita Valvassori (Universidad de Los Lagos)

El modelo narrativo del Decamerón en la Edad de Oro: una vieja historia .21

Antonio Gargano (Università degli Studi di Napoli Federico II)

«Difficile est proprie communia dicere»: el género de la novella entre

Boccaccio y Cervantes

Guillermo Carrascón (Università degli Studi di Torino)

Apuntes para un estudio de la presencia de Bandello en la

novela corta del siglo XVII

Leonardo Coppola (Università degli Studi «G. d'Annunzio» di Chieti-Pescara)

La proyección de Straparola en la novela española del Siglo de Oro desde una perspectiva editorial

Mireia Aldomì García

Didactismo, género literario y lector en Giraldi Cinzio.

María Jesús Zamora (Universidad Autónoma de Madrid)

«...En tiempo menos discreto que el de agora, aunque de hombres más sabios, se

Ilamaban a las novelas cuentos». La novela corta y el cuento en el Siglo de Oro.....109

Marcial Rubio (Università degli Studi «G. d'Annunzio» di Chieti-Pescara)

La contribución de Cervantes a la novela barroca: la ejemplaridad. .125

PIERRe Darnis (Université Bordeaux Montaigne)

La fuerza de la sangre, La ilustre fregona $y$ Las dos doncellas: ¿tres tipos

folclóricos?

María Soledad ArRedondo (Universidad Complutense de Madrid)

De La gitanilla $a$ La sabia Flora malsabidilla. El género, el personaje

y el matrimonio

Antonella Gallo (Università degli Studi di Verona)

Fabulaciones en equívocos burlescos: la Chrónica del monstro imaginado (1615)

de Alonso de Ledesma y novela corta barroca

David GonZález Ramírez (Universidad de Málaga)

El filósofo del aldea (1625) de Baltasar Mateo Velázquez: recepción textual

e hipótesis autorial.

Jonathan BRAdBury (University of Exeter)

La narrativa breve en la miscelánea del siglo XVII 
Cristina Castillo Martínez (Universidad de Jaén)

«La fuente del desengaño»: de las Noches de invierno de Eslava a la Tercera

Diana de Tejeda.

María Zerari (Université Paris-Sorbonne, CLEA)

Furor in fabula: La cruel aragonesa de Castillo Solórzano (o de la dama monstruo).. 241

Giulia Giorgi (Università degli Studi di Ferrara)

Alonso de Castillo Solórzano reescritor de sí mismo: algunas notas sobre los

Escarmientos de amor moralizados y el Lisardo enamorado .257

Angela Fabris (Alpen-Adria-Universität Klagenfurt)

El diálogo con el público y los espacios reales y de maravilla en

Casos prodigiosos y cueva encantada de Juan de Piña .267

María Rocío LePe García (IES San Sebastián, Huelva)

La traducción inglesa de Hipólito y Aminta: una adaptación

con fines comerciales 281

Andrea Bresadola (Università degli Studi di Udine)

La novela española en la Italia del siglo XVII: el caso de Il Feniso

de Francisco de Quintana

José Teruel (Universidad Autónoma de Madrid)

El triunfo del Desengaño. Marco y desengaño postrero de la Parte segunda

del Sarao y entretenimiento honesto, de María de Zayas

Nieves Romero-Díaz (Mount Holyoke College)

Lecturas alternativas en la Novela del fin bueno en mal principio

de doña Ana Francisca Abarca de Bolea.

Shifra Armon (University of Florida)

Compromiso y distanciamiento en La Venus de Ferrara

de Mariana de Carvajal Saavedra

Mechthild Albert (Rheinische Friedrich-Wilhelms-Universität Bonn)

Las "noches": un subgénero novelístico en perspectiva comparada.... .365

Fernando Copello Jouanchin (Université du Maine, Le Mans)

El mueble en la novela corta del Siglo de Oro: algunas reflexiones

en torno a la cama

Ilaria Resta (Università del Salento):

De la novella al entremés pasando por la novela corta: reescrituras del cuento

La gara delle tre mogli del Cieco di Ferrara. 


\section{EDAD DE ORO}

Revista de Filología Hispánica XXXIII

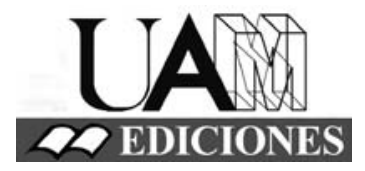




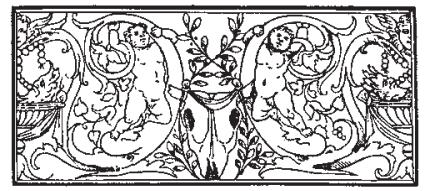

\section{Edad de Oro. Revista de Filología Hispánica}

ISSN: 0212-0429

Dirección:

Teodosio Fernández

Secretaría y edición:

José Ramón Trujillo

Coordinador del volumen XXXIII:

Rafael Bonilla Cerezo

Comité científico internacional:

Carlos Alvar (Univ. de Ginebra)

Ignacio Arellano (Univ. de Navarra)

Javier Blasco (Univ. de Valladolid)

Alberto Blecua (UAB)

Jean Canavaggio (Univ. de París X)

Laura Dolfi (Univ. de Turín)

Aurora Egido (Univ. de Zaragoza)

Víctor García de la Concha (RAE)

Luciano García Lorenzo (CSIC)

Joaquín González Cuenca (Univ. de Castilla-

La Mancha)

Agustín de La Granja (Univ. de Granada)

Begoña López Bueno (Univ. de Sevilla)

Michel Moner (Univ. de Toulouse III)

Joan Oleza (Univ. de Valencia)

Alfonso Rey (Univ. de Santiago)

Lina Rodríguez Cacho (Univ. de Salamanca)

Leonardo Romero Tobar (Univ. de Zaragoza)

Aldo Ruffinatto (Univ. de Turín)

Lía Schwartz (City University of New York)
Redacción y admisión de originales:

Teodosio Fernández

Edad de Oro

Departamento de Filología Española

Universidad Autónoma de Madrid

28049 Madrid (España)

Tfno.: +0034 914974090

correo: teodosio.fernandez@uam.es

Distribución, suscripción y venta:

Servicio de Publicaciones de la UAM

Universidad Autónoma de Madrid

28049 Madrid (España)

Intercambio de publicaciones:

Biblioteca de la Facultad de Filosofía y

Letras (UAM)

Universidad Autónoma de Madrid

28049 Madrid (España)

Han colaborado en este volumen:

Departamento de Filología Española (UAM)

Facultad de Filosofía y Letras (UAM)

Proyecto I+D FFI2013-41264-P La novela

corta del siglo XVII: estudio y edición (y II)

Edad de Oro se recoge en las siguientes bases de datos: SCOPUS, MLA Database, HLAS, Latindex, PIO-Periodical Content Index, ISOC, Dialnet, MIAR, ERIH, DICE, Sumaris CBUC, Ulrich's. Se encuentra evaluada en CIRC: A; INRECH: primer cuartil, posición 6 de 50; MIAR difusión ICDS live: 9.977; SCImago Journal \& Country Rank: H Index 2, SJR 0,101, Q4; RESH índice de impacto: 0.162; ERIH: A INT1; Carhus Plus+: B. 